\title{
Trichloroacetic acid assisted synthesis of gold nanoparticles and its application in detection and estimation of pesticide
}

\author{
Gadadhar Barman, Swarnali Maiti and Jayasree Konar Laha*
}

\begin{abstract}
Background: Many analytical methods are available for detection of methyl parathion in water but they are not handy for on-site analysis. An attempt has been made to utilize stable GNP for methyl parathion detection by sensing the peak at $400 \mathrm{~nm}$ generated due to the interaction between methyl parathion and GNP.

Methods: GNP was produced by reduction of chloroauric acid solution by trichloroacetic acid in alkaline medium in presence of CTAB. Sensor properties of GNP were studied by varying the concentration of methyl parathion in gold sol from 0 to 500 ppm.

Results and discussion: GNP stabilized by CTAB showed only one peak at $532 \mathrm{~nm}$ and one broad peak near 300 $\mathrm{nm}$ was observed for pure methyl parathion. But as soon as methyl parathion was added in the GNP solution, one new peak at $400 \mathrm{~nm}$ developed in addition to the other two peaks. More interestingly, a quantitative decrease of the absorbance at $532 \mathrm{~nm}$ of GNP and increase of the absorbance at $400 \mathrm{~nm}$, the new peak, were observed when methyl parathion concentration increased from 10 to 500 ppm.
\end{abstract}

Conclusions: The UV-VIS measurement and TEM images confirmed that the surfactant capped GNP can act as a colorimetric sensor for detection and estimation of methyl parathion pesticide present in water in ppm level.

Keywords: Gold nanoparticles, Cetyl trimethyl ammonium bromide, Sensor, Detection of methyl parathion, Estimation of pesticide, Spectroscopy

\section{Background}

The exponential growth in research in gold nanoparticles has been mainly due to the following reasons (i) stability of gold nanoparticles, (ii) many relatively easy preparation methods, (iii) its role in nanoscience and nanotechnology. For preparation of gold nanoparticles, many different reducing agents and stabilizing compounds have been employed to control shape and size of the particles. When gold nanoparticles approach each other and aggregate, the colour changes from red to blue because of the shift of the surface plasmon band to longer wavelength. The distinctive colors of gold nanoparticles have inspired people for years to use them as sensors because of several advantages over conventional electrochemical sensors. The literature

\footnotetext{
* Correspondence: j.laha@yahoo.co.in

Department of Chemistry, Midnapore College, Midnapore 721101, W.B, India
}

shows that the use of conventional analytical methods for detection of pesticide is getting replaced by sensors due to ease of application. The detection of pesticide residues is done using different biosensors (Airoldi et al. 2007). Several enzymes have been used in the development of electrochemical biosensor based on the inhibition mode of the enzyme for the determination of pesticides (Upadhyay et al. 2009). GNP based dipstick immunoassay was developed for the rapid detection of organochlorine pesticides such as DDT at nanogram level (Lisa et al. 2009). Along similar lines, in the present work we have developed a method for preparation of gold nanoparticles (GNP) using trichloroacetic acid as reducing agent in alkaline medium in presence of surfactant which acts as capping agent. This surfactant capped GNP has been used as colorimetric sensor for detection and estimation of pesticide (methyl parathion) present in a sample. Methyl parathion is chosen because it is a 
highly neurotoxic agricultural chemical that is used extensively worldwide to control a wide range of insect pests. Its residue in the soil causes pollution in the environment and poses a risk to human health. We have employed cetyl trimethyl ammonium bromide (CTAB) capped GNP as sensor and the change in UV-Visible spectra was monitored when pesticide (methyl parathion) was added at ppm level.

Many analytical methods are available for detection of methyl parathion in water. The common analytical techniques include HPLC (high performance liquid chromatography) with UV detection (Huang et al. 2002), GC-Mass spectrometry (Ferrer et al. 2005; Garcia-Reyes et al. 2007), GC/ECD (gas chromatography coupled with electron capture) (Bicchi et al. 2003; Brito et al. 2002), GC/FPD (gas chromatography coupled with flame photometry) (Berijani et al. 2006), GC/FID (flame ionization detection) (Pinheiro \& Andrade 2009). All these methods are very sensitive but each one requires its unique method for sample preparation and above all, they are not handy for on-site analysis. These are mainly applied in laboratory settings and restrict rapid analysis under field conditions. At the same time they are expensive and time consuming. For this reason, an attempt has been made to utilize stable GNPs for methyl parathion detection. The nano colloidial GNPs could detect methyl parathion by forming bond using sulphur present in methyl parathion and thereby generating a new peak near $400 \mathrm{~nm}$. The height of this peak is proportional to the concentration of methyl parathion and this makes for a ready means of estimating the pesticide concentration.

\section{Methods}

\section{Preparation}

Trichloroacetic acid, chloroauric acid, cetyl trimethyl ammonium bromide, all AR grade, were purchased from Sigma-Aldrich Chemical Ltd. Sodium hydroxide and methyl parathion were purchased from Merck. Double distilled de-ionized water was used in all experiments.

GNP was produced by reduction of chloroauric acid solution by trichloroacetic acid in alkaline medium in presence of $\mathrm{CTAB}$. The $\mathrm{pH}$ was varied during reduction by adding different amounts of $\mathrm{NaOH}$ solution. $10 \mathrm{ml}$ of $3 \times 10-3(\mathrm{M})$ trichloroacetic acid solution was added to an equal volume of the same concentration of alkaline CTAB. The mixture was cooled in ice cold water. Then $7 \mathrm{ml}$ of $3 \times 10-3(\mathrm{M})$ aqueous chloroauric acid was added drop wise with continuous stirring. The mixture was cooled for 10 minutes and then it was heated at $850 \mathrm{C}$ for 1 hour. The colour of the solution gradually changed from yellow to violet to reddish violet. The reddish violet colour indicated the formation of gold nanoparticles (GNPs).

\section{Characterization}

The absorbance spectra of the GNPs were analyzed by using a 'SHIMADZU' UV 1800 spectrophotometer and TEM images were taken using JEOL-JEM 2100 high resolution transmission electron microscope (HR-TEM). Samples for the TEM studies were prepared by placing a drop of the aqueous suspension of particles on carboncoated copper grids followed by solvent evaporation under vacuum. The crystalline nature of the GNPs was examined using $X^{\prime}$ Pert-PRO model (Analytical Holland) $\mathrm{X}$-ray diffractometer.

Sensor properties of GNP were studied by varying the concentration of methyl parathion in gold sol from 0 to $500 \mathrm{ppm} .250 \mu \mathrm{l}$ of solution containing different concentrations of methyl parathion was added to $5 \mathrm{ml}$ of "as prepared" GNP. The sol was heated for 5 minutes with stirring. The reddish violet color changed into yellow. The intensity of yellow colour gradually increased with increase of pesticide concentration.

\section{Results and discussion Optimization of GNP preparation}

GNP displays optical properties due to the presence of surface plasma resonance (SPR) band. For spherical gold particles the SPR occurs nearly at $540 \mathrm{~nm}$. Small shifts in the SPR band occur perhaps due to the changes in the dielectric properties of the medium of the GNP or due to the specific adsorption of materials on the surface of the gold particle. Mie theory is generally used to explain the shifts in the SPR band (Mie 1908; Creighton \& Eadon 1991). According to this theory, shifts in SPR may also occur when particles geometry changes from spherical to some other shapes. In the present work, the GNP as prepared produces SPR band at $532 \mathrm{~nm}$. We have observed that the $\mathrm{pH}$ and concentration of the surfactant have important roles in the formation of GNP. Hence the effects of $\mathrm{pH}$ and concentration of CTAB were investigated thoroughly. To optimize the $\mathrm{pH}$ of the solution it was varied from 7.0 to 11.0 during the reaction. When the $\mathrm{pH}$ is higher than 8.0 and lower than 10.5, the GNP solution was stable for more than six months. However, if the $\mathrm{pH}$ is lower than 8.0, the precipitation occurred within several hours. One interesting observation should be pointed out that though $\mathrm{pH}$ and CTAB concentration have effect on the stability of the GNP formation yet the SPR band was fixed at $532 \mathrm{~nm}$ in all cases. From the extinction spectra (Figure 1A) it is seen that maximum absorbance of GNP peak i.e. $532 \mathrm{~nm}$ peak is found at $\mathrm{pH}$ 9.5. Furthermore, it has been observed that $3 \times 10-3$ (M) concentration of surfactant produced most stable sol, though $5 \times 10-3(\mathrm{M})$ concentration produced higher extinction coefficient of $532 \mathrm{~nm}$ peak (see Additional file 1: Figure $\mathrm{S} 1 \mathrm{~B})$. At higher concentration of the surfactant, precipitation of the sol occurred. That is why in our experiments we 

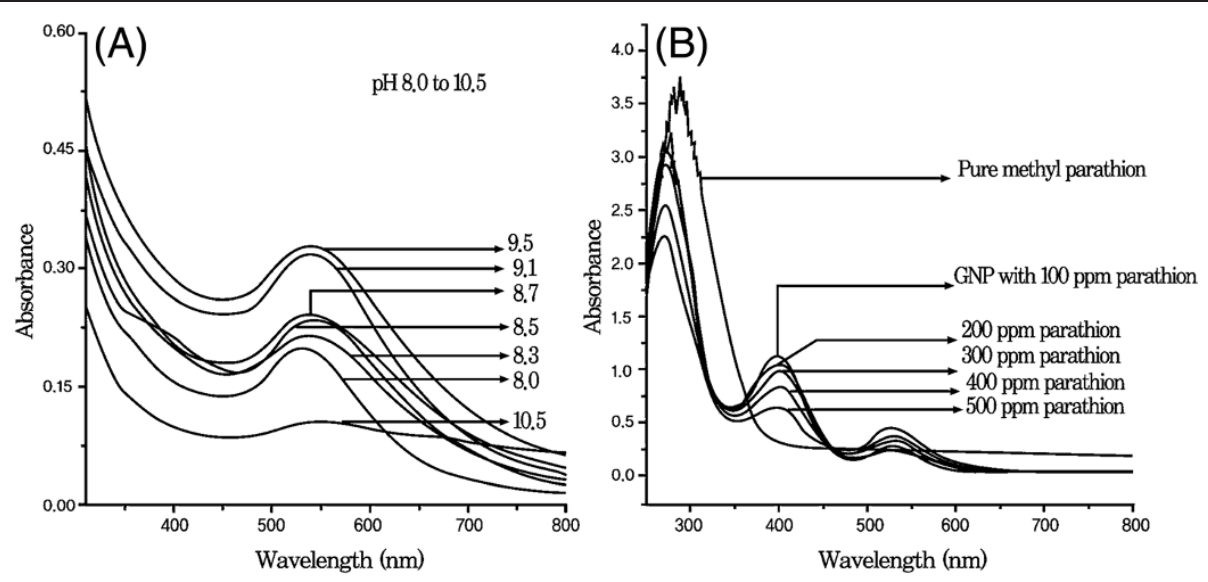

Figure 1 UV-vis spectra of (A) GNP at different $\mathrm{pH}$ and (B) Pure methyl parathion and GNP with different concentrations of methyl parathion.
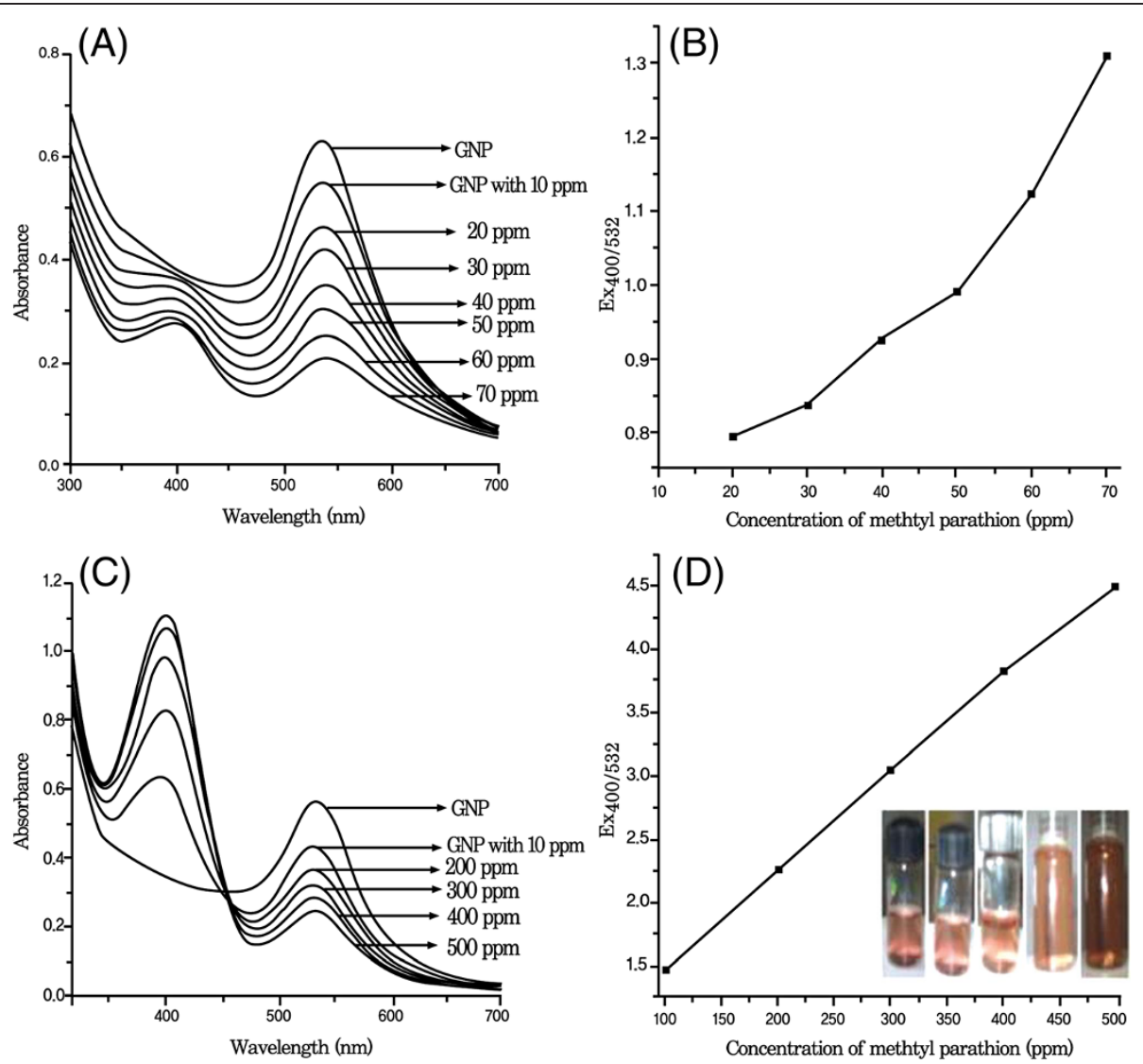

Figure 2 UV-vis spectra of GNP and GNP with various concentrations of methyl parathion (A) 10 to $70 \mathrm{ppm}$ (C) 100 to $500 \mathrm{ppm}$ and corresponding changes of absorption coefficients (Ex 400/532) with various concentrations of methyl parathion (B) 10 to 70 ppm (D) 100 to 500 ppm. 2(D) (inset): digital photographic images of color changes due to addition of methyl parathion. 

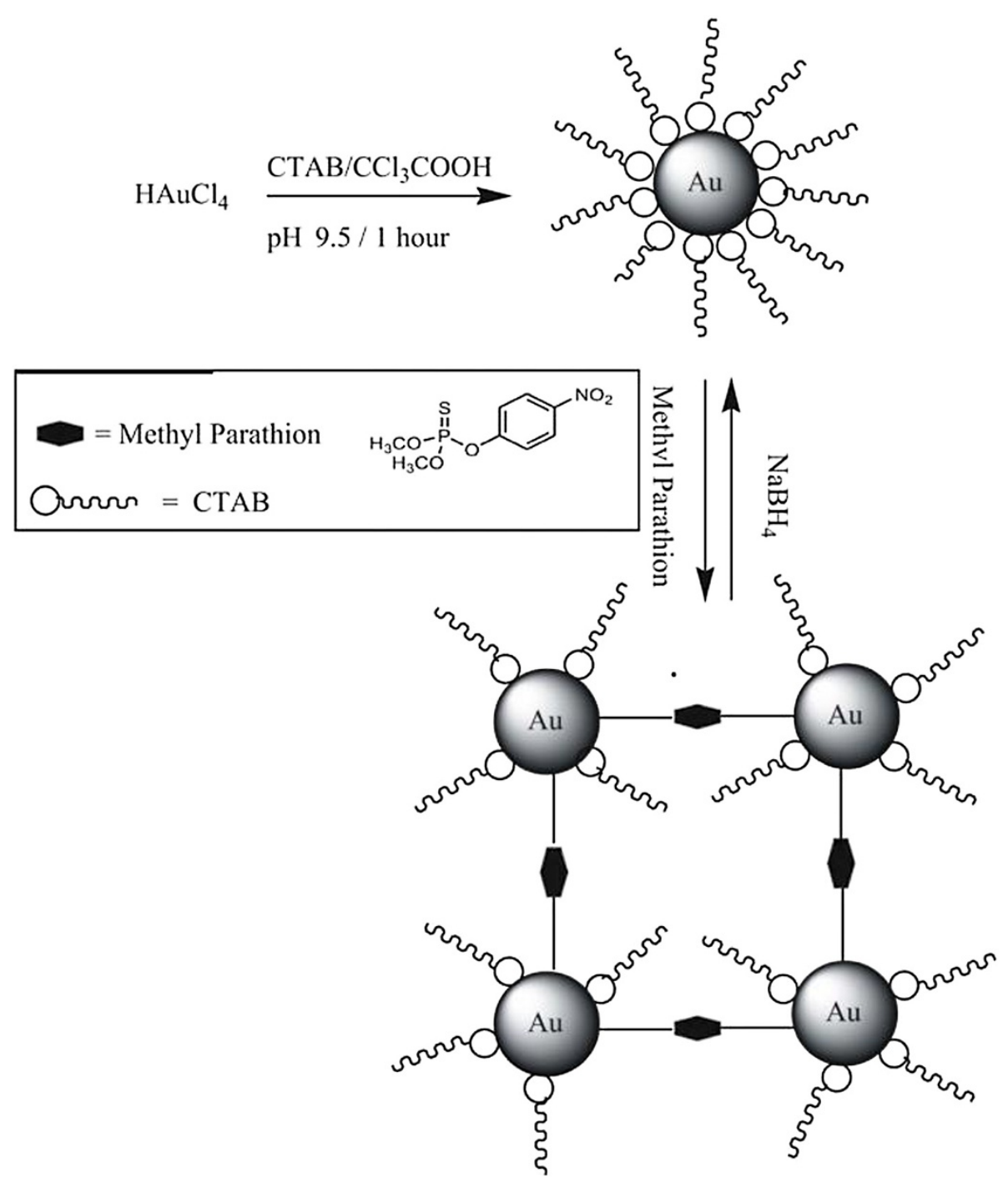

Scheme 1 Strategy for the formation, aggregation and segregation of GNP.

have used $3 \times 10-3(\mathrm{M}) \mathrm{CTAB}$ with equi-molar chloroaruric acid at $\mathrm{pH} 9.5$ to get most stable GNP.

The change in the absorbance spectra due to the increasing methyl parathion concentration from 0 to $\mathrm{C}$ depicted in Figure 1(B), 2(A) \& (C).

GNP stabilized by CTAB shows only one peak at $532 \mathrm{~nm}$ while pure methyl parathion shows one broad peak near $300 \mathrm{~nm}$. But as soon as methyl parathion is added in the GNP solution, one new peak at $400 \mathrm{~nm}$ starts developing in addition to the other two peaks. More interestingly, a quantitative decrease of the absorbance peak at $532 \mathrm{~nm}$ of GNP and increase of the absorbance at $400 \mathrm{~nm}$, the new peak, are observed when methyl parathion concentration increases from 10 to 500 ppm. The appearance of the new peak near $400 \mathrm{~nm}$ might be due to the bond formation between phosphorothioate group present in methyl parathion and the GNP. A control experiment shows that no such peak is obtained when methyl parathion is added in pure chloroauric acid (see Additional file 1: Figure S2B). It is well known that many compounds containing sulphur are specifically being employed for functionalization of GNP through ligand exchange reaction.

In recent years, mercaptopropionic acid (Huang \& Chang 2007), mercaptoundecanoic acid (Templeton et al. 1998), glutathione (Chai et al. 2010), etc. were used for this purpose. From the chemical structure of the pesticide we can also predict that phosphorothioate group may definitely help the functionalization of GNP due to which the new peak appears near $400 \mathrm{~nm}$ (Figure 1(B), 2(A) \& 2(C)). 
The probable mechanism for bond formation and aggregation of sol particles are elaborated in Scheme 1.

The absorption coefficient ratio (the ratio of absorption coefficient of 400 and $532 \mathrm{~nm}$ peaks, Ex 400/532), which measures the changes in absorption peaks due to the addition of various concentrations of pesticide, has been investigated thoroughly. The ratio increases with the increase of concentration of the pesticide. This phenomenon suggests that more the phosphorothioate group more is the functionalization of GNP up to a certain concentration of pesticide. A calibration curve between the absorption coefficient ratios versus concentration of pesticide can help one to estimate quantitatively the presence of methyl parathion in a sample at ppm level (Figure 2(B) \& 2(D)).

The TEM measurements of GNP produced at around pH 9.5 show almost spherical particles of different sizes (Figure 3(A)). The capping of the hydrophobic part of CTAB on GNP shows a layer surrounding the gold particle. In presence of methyl parathion, the size, shape altogether changes drastically and a completely different look with an exciting feature is observed (Figure 3(B), 3(C) \& 3(D)).

It appears that restructuring of GNP occurs after addition of methyl parathion. Spherical particles become spheroidal and agglomeration of particles is observed. It is likely that the surface of the GNP forms an Au-S co-ordination bond (this can happen as the sol is being heated after addition of methyl parathion) and some methyl parathion molecules get adsorbed on the Au surface by replacing CTAB. As methyl parathion is anionic in alkaline medium, its adsorption on the GNP surface lowers the surface charge and thus they agglomerate and the interesting particle clustering is observed. Each cluster has an average size of $50 \mathrm{~nm}$. These clusters segregate to give back GNP reversibly when reduction is performed with $\mathrm{NaBH}_{4}$ (Scheme 1 and Figure 4).

Detailed studies are in progress to know the exact chemical nature of the bond formed between GNP and methyl parathion. A recent review discusses the recent progress in understanding of molecular structure of the gold-sulfur interface in thiolate-protected gold surfaces and interfaces from the viewpoint of theory and computations, with connections to relevant experiments (Hakkinen 2012). Though thiolate group is not present in this work, phosphorothioate group may be responsible for the Au-S interaction. We are also interested to know what sort of interactions is actually taking place on the GNP surface with methyl parathion so that new peak develops at $400 \mathrm{~nm}$. To investigate this bonding XRD and FTIR have been employed. The XRD
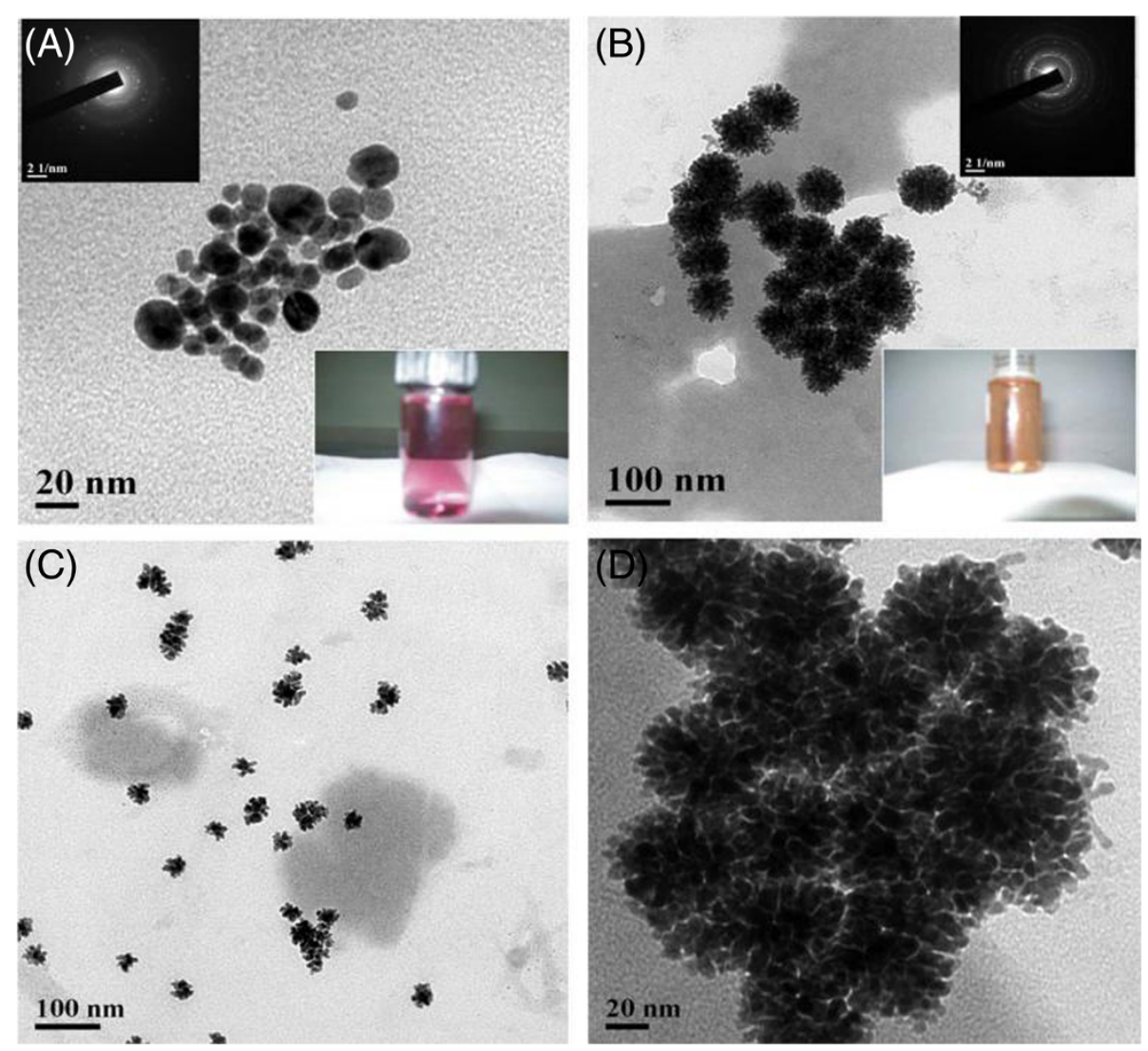

Figure 3 TEM micrographs of GNP (A) before and (B), (C) \& (D) after addition of methyl parathion. 


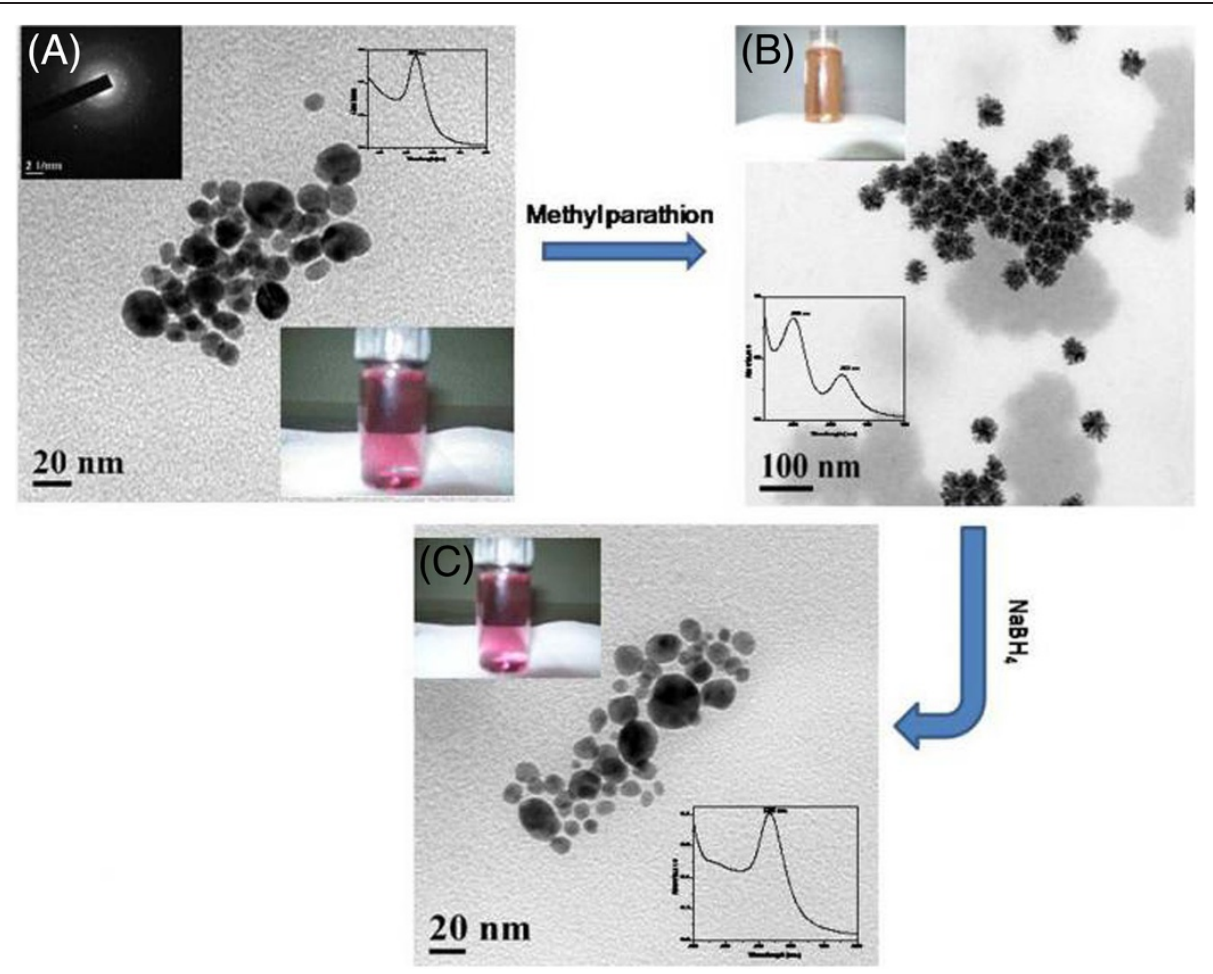

Figure 4 A strategy of aggregation of GNP (A) due to reaction with methyl parathion (B) and segregation of GNP by reduction with $\mathrm{NaBH}_{4}(\mathrm{C})$.

pattern of the GNP shown in Figure 5(A) indicates the face centered cubic (fcc) structure of the bulk gold having peaks at $38.175 \mathrm{o}, 44.525 \mathrm{o}, 64.675 \mathrm{o}, 77.675 \mathrm{o}$ and $81.825 \mathrm{o}$ corresponding to (111), (200), (220), (311) and (222) planes, respectively. In literature we find $\mathrm{Au} 2 \mathrm{~S}$ shows peaks near 31o, 35.5o, 51.5o, 61.5o and 65o in XRD (Kuo \& Huang 2008). The XRD spectrum of the GNP after reaction with methyl parathion are shown in Figure 5(B) and it is visible that the spectrum has all peaks due to Au as well as some additional peaks at $50.5 \mathrm{o}, 60.060$ and $64.5 \mathrm{o}$ which may be attributed to the Au-S co-ordination bond.

FTIR spectra (Additional file 1: Figure S5) of the GNP before and after addition of methyl parathion show almost no changes in the peak positions (slightly shifted). The peaks are mainly due to the CTAB present in the system (Liu et al. 2007). The comparison of the spectra shows the appearance of new peaks near $1480 \mathrm{~cm}-1$ and $853 \mathrm{~cm}-1$ which we are unable to assign. More work is needed to understand the
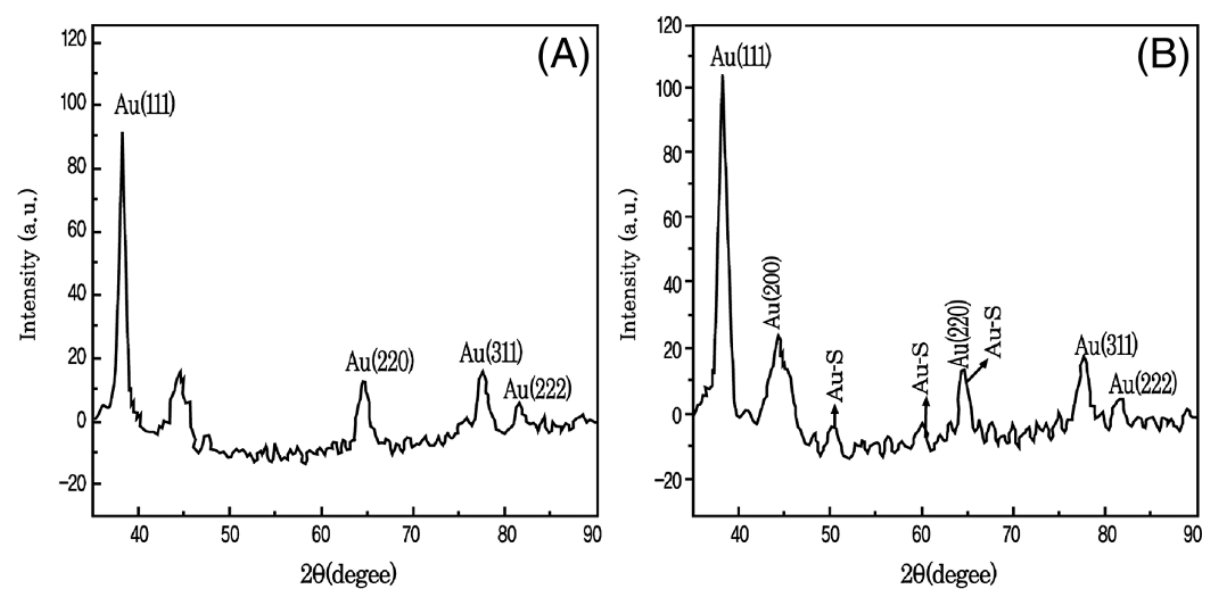

Figure 5 XRD of GNP (A) before and (B) after addition of methyl parathion. 
nature of the bond which may be crucial for some applications of gold based nanoparticles for some other purposes.

\section{Conclusion}

We have employed CTAB capped GNP as sensor and the change in UV-Visible spectra was monitored when methyl parathion was added at ppm level. The UV-VIS measurements and TEM images confirm that the surfactant capped GNP can act as a colorimetric sensor for detection and estimation of methyl parathion pesticide present in ppm levels by utilizing the bonding between GNP and phosphorothioate group of the pesticide. Agglomeration of the GNPs occurs due to the adsorption of methyl parathion on the GNP surface, and hence the interesting particle clustering is observed. These clusters segregate to give back GNP reversibly when reduction with $\mathrm{NaBH}_{4}$ is done.

\section{Supporting information}

UV-vis spectra of GNP formation with varying time and concentrations of chloroauric acid and CTAB result of control experiment with pure chloroauric acid, strategy and some TEM photographs for aggregation and segregation of GNP. These materials are available free of charge on the Web at http://www.jsac.or.jp/analsci/.

\section{Additional file}

Additional file 1: Figure S1. UV-Vis spectra of GNP formation with varying concentrations of (A) chloroauric acid (B) CTAB. Figure S2. UV-Vis spectra of GNP formation varying time of reaction from 20 minutes to 2 hours keeping chloroauric acid concentration constant at $3 \times 10-3$ (M) and (B) Absorption spectra of pure chloroauric acid in presence of methyl parathion at various concentration. Figure S3. Different TEM micrographs of GNP (A) \& (B) and GNP after reaction with methyl parathion (C) \& (D). Figure S4. UV representation of aggregation of GNP (A) due to reaction with methyl parathion (B) and segregation of GNP by reduction with $\mathrm{NaBH}_{4}$ (C). Figure S5. FTIR spectra of (A) GNP and (B) GNP with methyl parathion.

\section{Competing interests}

The authors declare that they have no competing interests.

\section{Authors' contributions}

GB carried out the experiment. GB and SM drafted the manuscript. JKL guided the research and modified the manuscript. All three authors read and approved the final manuscript.

\section{Authors' information}

JKL is Associate Professor and Head of Department of Chemistry, Midnapore College, West Bengal, India. GB and SM are research scholars of this Department.

\section{Acknowledgements}

We gratefully acknowledge the financial support received from UGC (Ref.No. F. PSW-096 / 10-11.) We are also thankful to Central Research Facility at IIT Kharagpur, India for HR-TEM and XRD measurements.

\section{References}

Airoldi FPS, Da Silva WTL, Crespilho FN, Rezende MOO (2007) Evaluation of the Electrochemical Behavior of Pentachlorophenol by Cyclic Voltammetry on Carbon Paste Electrode Modified by Humic Acids. Water Environ Res 79:63

Berijani S, Assadi Y, Anbia M, Hosseini MRM, Aghaee E (2006) Dispersive liquid-liquid microextraction combined with gas chromatography-flame photometric detection: Very simple, rapid and sensitive method for the determination of organophosphorus pesticides in water. J Chromatogr. A. 1123:1

Bicchi C, Cordero C, lori C, Rubiolo P, Sandra P, Janete H, Yariwake JH, Zuin VG (2003) SBSE-GC-ECD/FPD in the Analysis of Pesticide Residues in Passiflora alata Dryander Herbal Teas. J Agric Food Chem 51:27

Brito NM, Navickiene S, Polese L, Jardim EFG, Abakerli RB, Ribeiro ML (2002) Determination of pesticide residues in coconut water by liquid-liquid extraction and gas chromatography with electron-capture plus thermionic specific detection and solid-phase extraction and high-performance liquid chromatography with ultraviolet detection. J Chromatogr. A. 957:201

Chai F, Wang C, Wang T, Li L, Su Z (2010) Colorimetric detection of $\mathrm{Pb}^{2+}$ using glutathione functionalized gold nanoparticles. ACS Appl Mater Interfaces 25:1466

Creighton JA, Eadon DG (1991) Ultraviolet-visible absorption spectra of the colloidal metallic elements J. Chem. Soc. Faraday Trans. 87:3881

Ferrer I, Thurman EM, Fernandez-Alba AR (2005) Quantitation and accurate mass analysis of pesticides in vegetables by LC/TOF-MS. Anal Chem 77:2818

Garcia-Reyes JF, Hernando MD, Ferrer C, Molina-Diaz A, Fernandez-Alba AR (2007) Large Scale Pesticide Multiresidue Methods in Food Combining Liquid Chromatography, Time-of-Flight Mass Spectrometry, and Tandem Mass Spectrometry. Anal Chem 79:7308

Hakkinen H (2012) The Gold-Sulfur Interface at the Nanoscale. Nature Chem. 4:443

Huang CC, Chang HT (2007) Parameters for selective colorimetric sensing of mercury (II) in aqueous solutions using mercaptopropionic acid-modified gold nanoparticles. Chem Commun 12:1215

Huang G, Ouyang J, Baeyens WRG, Yang Y, Tao C (2002) High-performance liquid chromatographic assay of dichlorvos, isocarbophos and methylparathion from plant leaves using chemiluminescence detection. Anal Chim Acta 474:21

Kuo CL, Huang MH (2008) Hydrothermal Synthesis of Free-Floating Au2S Nanoparticle Superstructures. J Phys. Chem. C. 112:11661

Lisa M, Chouhan RS, Vinayaka AC, Manonmani HK, Thakur MS (2009) Gold nanoparticles based dipstick immunoassay for the rapid detection of dichlorodiphenyltrichloroethane: An organochlorine pesticide. Biosens Bioelectron 25:224

Liu XH, Luo XH, Lu SX, Zhang JC, Cao WL (2007) A novel cetyltrimethyl ammonium silver bromide complex and silver bromide nanoparticles obtained by the surfactant counterion. J Colloid Interface Sci. 307:94

Mie G (1908) Contributions to the Optics of Turbid Media, Especially Colloidal Metal Solutions. Ann. Physik. 25:377

Pinheiro AS, Andrade JB (2009) Development, validation and application of a SDME/GC-FID methodology for the multiresidue determination of organophosphate and pyrethroid pesticides in water. Talanta 79:1354

Templeton AC, Hostetler MJ, Warmth EK, Chen SW, Hartsshorn CM, Krishnamurty VM, Forbes MDE, Murray RW (1998) Gateway Reactions to Diverse, Polyfunctional Monolayer-Protected Gold Clusters, J Am. Chem. Soc. 120:4845

Upadhyay S, Rao GR, Sharma MK, Bhattacharya BK, Rao VK, Vijayaraghavan R (2009) Immobilization of acetylcholineesterase-choline oxidase on a goldplatinum bimetallic nanoparticles modified glassy carbon electrode for the sensitive detection of organophosphate pesticides, carbamates and nerve agents. Biosens Bioelectron 25:832

\section{doi:10.1186/2093-3371-4-3}

Cite this article as: Barman et al:: Trichloroacetic acid assisted synthesis of gold nanoparticles and its application in detection and estimation of pesticide. Journal of Analytical Science and Technology 2013 4:3. 